\title{
Tradução, adaptação e validação do Relationship Questionnaire em jovens portugueses
}

\author{
Translation, adaptation and validation of the Relationship Questionnaire among portuguese \\ young people \\ Traducción, adaptación y validación del Relationship Questionnaire en jóvenes portugueses
}

Maria dos Anjos Coelho Rodrigues Dixe*; Helena da Conceição Borges Pereira Catarino**; Susana Margarida Rodrigues Custódio***; Cristina Maria Figueira Veríssimo****; Joana Alice da Silva Amaro Oliveira Fabião ${ }^{* * * *}$; Maria da Conceição Gonçalves Marques Alegre de Sá******

\section{Resumo}

Enquadramento: No âmbito das relações interpessoais, os relacionamentos amorosos assumem um significado especial no final da adolescência.

Objetivos: Este estudo metodológico teve como principal objetivo traduzir e adaptar para a cultura portuguesa o Questionário do Relacionamento Amoroso.

Metodologia: Participaram 127 estudantes do ensino superior, selecionados por amostragem não probabilística intencional, tendo sido aplicado um questionário (questões relativas a características sociodemográficas e de relacionamento amoroso e o questionário de relacionamento amoroso).

Resultados: Os resultados apontam para uma escala de 16 itens, distribuídos por 4 fatores (Relação possessiva, Relação controlo, Relação depreciativa e Relação destrutiva), que explicam $63,850 \%$ da variância. A matriz de correlação $\left(\chi^{2}=840,544 ; \mathrm{p}<, 000\right)$ e a medida de Kaiser-Meyer-Olkin $(\mathrm{KMO}=0,815)$ garantem a adequação do modelo fatorial. Apresenta uma boa consistência interna ( $\alpha$ de Cronbach de 0,847 ). Os dados confirmam o instrumento como rigoroso, fiável e válido. Os jovens apresentam uma qualidade de relacionamento saudável, sendo este relacionamento melhor nas raparigas que nos rapazes.

Conclusão: Os resultados indiciaram estarmos perante um questionário fiável e válido para avaliar o relacionamento amoroso.

Palavras-chave: jovem; relacionamento amoroso, psicometria e medidas.

\begin{abstract}
Background: Within interpersonal relationships, romantic relationships take on a special meaning at the end of adolescence.

Objectives: The main purpose of this methodological study was to translate and adapt the Relationship Questionnaire into the Portuguese culture. Methodology: A questionnaire (questions related to socio-demographic characteristics and romantic relationships, as well as the relationship questionnaire) was applied to a sample composed of 127 higher education students, obtained using a non-probability purposive sampling.

Results: Results showed a 16-item scale, distributed into four factors (Possessive relationship, Controlling relationship, Demeaning relationship, and Destructive relationship), which explained $63.850 \%$ of the variance. The correlation matrix $\left(\chi^{2}=840.544 ; \mathrm{p}<.000\right)$ and the Kaiser-Meyer-Olkin measure $(\mathrm{KMO}=0.815)$ ensured the adequacy of the factor model. The scale showed good internal consistency (Cronbach's alpha $=0.847$ ). Data confirmed that the instrument is accurate, reliable and valid. Young people have healthy relationships, though female students have better relationships than male students.

Conclusion: Results indicated that this is a reliable and valid questionnaire to assess romantic relationships.
\end{abstract}

Keywords: young person; romantic relationship; psychometrics and measures.

\footnotetext{
* PhD, RN, Professora Coordenadora, Escola Superior de Saúde - Instituto Politécnico de Leiria, Unidade de Investigaçāo em Saúde, 2411 - 901, Leiria, Portugal. Membro da equipa do projecto (O) Usar \& Ser Laço Branco [maria.dixe@ipleiria.pt]. Morada para correspondência: Instituto Politécnico de Leiria, Campus 2 Morro do Lena - Alto do Vieiro, Apartado 4137, 2411-901, Leiria, Portugal

* PhD, RN, Professora Coordenadora, Escola Superior de Saúde - Instituto Politécnico de Leiria, Unidade de Investigaç̃o em Saúde, 2411 - 901, Leiria, Portugal. Membro da equipa do projecto (O) Usar \& Ser Laco

Branco [helena.catarino@ipleiria.pt].
**** PhD, Professora Ajunta, Escola Superior de Saúde - Instituto Politécnico de Leiria, Unidade de Investigação em Saúde, 2411 - 901, Leiria, Portugal. Membro da equipa do projecto (O)Usar \& Ser Laço Branco. [susana.custodio@ipleiria.pt).

**** MPH, RN, Doutoranda em Enfermagem, Professora Adjunta, Escola Superior de Enfermagem de Coimbra, 3046-851, Coimbra, Portugal. Membro da equipa do projecto (O) Usar \& Ser Laço Branco

[cristina@esenfc.pt].
$* * * * *$ PhD, RN, Professora Ajunta, Escola Superior de Enfermagem de Coimbra, 3046-851, Coimbra Portugal. Membro da equipa do projecto (O)Usar \& Ser Laço Branco [jfabiao@esenfc.pt].

******** MPH, RN, Doutoranda em Enfermagem, Professora Adjunta, Escola Superior de Enfermagem de Coimbra 3046-851, Coimbra, Portugal. Membro da equipa do projecto (O)Usar \& Ser Laço Branco [calegre@esenfc.pt].
}

\section{Resumen}

Marco: En el ámbito de las relaciones interpersonales, las relaciones amorosas adquieren un significado especial al final de la adolescencia.

Objetivos: El objetivo principal de este estudio metodológico ha sido traducir y adaptar a la cultura portuguesa el Cuestionario de Relaciones Amorosas.

Metodología: Se contó con la participación de 127 estudiantes de enseñanza superior seleccionados por un muestreo no probabilístico intencional, y con un cuestionario (cuestiones relativas a las características sociodemográficas y de las relaciones amorosas, así como el cuestionario de relaciones amorosas). Resultados: Los resultados se reflejan en una escala de 16 ítems, distribuidos en 4 factores (relación posesiva, relación de control, relación de desprecio y relación destructiva), que explican el $63,850 \%$ de varianza. La matriz de correlación $\chi^{2}=840,544$; $\mathrm{p}<, 000)$ y la medida de Kaiser-Meyer-Olkin $(\mathrm{KMO}=0,815)$ garantizan la adecuación del modelo factorial. Asimismo, presenta una buena consistencia interna ( $\alpha$ de Cronbach de 0,847 ). Los datos confirman que el instrumento es riguroso, fiable y válido. Las relaciones entre los jóvenes son por tanto sanas, aunque la calidad de estas es mejor en las chicas que en los chicos.

Conclusión: Los resultados indican que nos encontramos ante un cuestionario fiable y válido de evaluación de las relaciones amorosas.

Palabras clave: joven, relaciones amorosas, psicometría y medidas.

Recebido para publicação em: 30.07.13

Aceite para publicação em: 05.02 .14 


\section{Introdução}

Uma das tarefas de desenvolvimento com a qual os adolescentes/jovens se confrontam prende-se com o estabelecimento de relações interpessoais de qualidade, as quais terão influência no processo de construção da sua identidade, integridade, autonomia, autoconceito, autoestima e gestão emocional.

No âmbito das relações interpessoais importa destacar as relações amorosas. Nesta fase da vida, os adolescentes caracterizam-se por uma maior autonomia face à família, desenvolvem relações íntimas com pares do mesmo sexo e começam a namorar. $\mathrm{O}$ namoro e o estabelecimento de relações amorosas representam uma transição para a adultez sendo para muitos adolescentes manifestações do estado adulto (Hand \& Furman, 2009).

Os relacionamentos amorosos assumem maior significado no final da adolescência, começando os adolescentes a ensaiar os equilíbrios entre a construção da intimidade e da identidade. Collins, Welsh, e Furman (2009) constataram a existência de associações entre as relações românticas na adolescência e aspetos do desenvolvimento individual, tais como construir uma identidade pessoal, adaptar-se às mudanças nas relações familiares, envolver-se em relações harmoniosas com pares, ter sucesso escolar e desenvolver a sua maturidade sexual.

No que concerne às diferenças de sexo, as raparigas tendem a reportar níveis mais elevados na qualidade da relação com o par amoroso, nomeadamente no que respeita à intimidade e à satisfação (Connolly \& McIssac, 2011).

A investigação revela a existência de diferenças nas características das relações românticas adolescentes em função da idade (Adams, Laursen, \& Wilder, 2001). As relações amorosas, em fases iniciais, são sobretudo caracterizadas por níveis elevados de afiliação, que se traduzem na procura de proximidade física, na partilha de atividades e no companheirismo (Adams et al., 2001). Com o aumento da idade, verifica-se uma tendência para a procura de proximidade emocional, manifestada através da interdependência, da reciprocidade e da diversidade de atividades que ocorrem entre os parceiros, bem como através de interação social diária (Adams et al., 2001; Pinto, 2009). Markiewicz, Lawford, Doyle, e Haggart (2006) constataram que no início da adolescência os níveis de suporte pelos pares aumentam. Contudo, verifica- se um declínio dos mesmos na adolescência tardia, sendo que nesta fase o suporte é providenciado pelo par romântico.

A relação amorosa traduz um fenómeno no qual a independência e a admiração mútua resultam numa forma de entendimento e de compreensão do par romântico, tornando-se cada elemento da díade uma figura de vinculação. Nesta perspetiva, uma relação saudável seria aquela em que cada indivíduo tem a sua própria identidade e deseja fazer o bem à pessoa amada, sem esperar recompensa (Rosset, 2004).

Para Sangrador (como citado por Fiol, 2007) o amor pode entender-se como uma atitude (atitude positiva ou de atração relativamente a outra pessoa, que inclui uma predisposição para pensar, sentir e comportar-se de um certo modo relativamente a essa pessoa), uma emoção (sentimento ou paixão que inclui determinadas reações fisiológicas) ou um comportamento (cuidar da outra pessoa, estar com ela, atender às suas necessidades). Importa assim aprofundar o estudo das relações amorosas em termos de comportamentos, sentimentos, conceitos e motivações.

As relações amorosas tenderão a influenciar de modo positivo os indivíduos, indo ao encontro das suas expectativas, auxiliando na resolução dos seus problemas e promovendo a satisfação das suas necessidades (Pinto, 2009). Servem igualmente como fatores protetores da vitimização e da solidão (Woodhouse, Dykas, \& Cassidy, 2012). Apesar do estabelecimento de relações de namoro constituir uma tarefa de desenvolvimento dos adolescentes/ jovens, nem sempre se processa de modo positivo, gratificante e adequado tanto para o próprio como para o outro. Assim, poderão surgir problemas tais como: o isolamento social, a timidez e ansiedade social e a violência (Shulman \& Kipnis, 2001).

As relações de amor na adolescência/juventude podem variar na sua intensidade (ténue-extrema) e na qualidade (agradável-dolorosa). Madsen e Collins (2011) constataram que os adolescentes que tiverem menos parceiros na adolescência intermédia e que experienciaram relações de melhor qualidade, aquando jovens adultos evidenciavam relações caracterizadas pela negociação de conflitos tendo por base a satisfação mútua, o cuidado e a atenção ao outro. Pelo contrário, os adolescentes que tinham tido mais parceiros tendiam a estabelecer relações românticas pautadas por uma afetividade negativa. 
Estudos realizados nos últimos anos têm demonstrado que a violência nas relações amorosas é um fenómeno frequente e habitual, evidenciando que esta se inicia com frequência nas relações de namoro (MuñozRivas, Gómez, O'Leary, \& Lozano, 2007).

A agressão indireta face ao par romântico é usada mais frequentemente pelas raparigas, sendo as relações mediadas pelo ciúme (Arnocky, Sunderani, Miller, \& Vaillancourt, 2012).

Nas relações amorosas um dos sentimentos que tende a estar presente é o ciúme romântico. Se para muitos o ciúme pode representar uma manifestação de amor, de afeto, de zelo que uma pessoa revela por outra, ele é, na verdade, um sentimento que produz angústia em muitos parceiros e pode atingir formas doentias, com consequências nefastas para a saúde física e mental dos indivíduos envolvidos. Estes são os casos em que o ciúme se torna patológico, doentio, traduzindo-se numa obsessão descontrolada e descontroladora (Almeida, Rodrigues, \& Silva, 2008). O que aparece neste tipo de ciúme é um grande desejo de controlo total sobre os sentimentos e comportamentos do companheiro.

O ciúme geralmente surge quando um relacionamento diádico valorizado é ameaçado. Implica o exercício de uma certa restrição sobre o outro, porque o parceiro ciumento, de algum modo, interfere no comportamento do outro e na sua liberdade, tornando-se possessivo e controlador. $\mathrm{O}$ ciúme poderá ter na sua base uma insegurança afetiva devida à interferência de um rival e pode envolver sentimentos como medo, suspeição, desconfiança, angústia, ansiedade, raiva, rejeição, indignação, constrangimento e solidão, entre outros, dependendo de cada pessoa (Arnocky et al., 2012).

Numa relação afetada pelo ciúme, as pessoas, geralmente, são tratadas como objetos pelos próprios parceiros. Muitas anulam-se e, assim, perdem grande parte da sua identidade para serem o que o ciumento quer que sejam, tentando corresponder a todas as suas expectativas (Almeida et al., 2008).

As relações amorosas dos adolescentes/jovens deverão ser, desejavelmente, a tradução de uma relação de cuidado, de atenção, encerrando em si, simultaneamente, mudanças e desafios pessoais, sociais e relacionais (Pinto, 2009). Deste modo importa promover uma melhor compreensão acerca das experiências amorosas que estabelecem ao longo do tempo e das implicações das mesmas na sua saúde e bem-estar (Starr et al., 2012).

É necessário abordar a temática das relações amorosas e da atenção ao outro pensando na prevenção das relações de violência e maltrato, com a finalidade última de evitar relações dominantes e vitimizadoras. Para tal é necessário o desenvolvimento de instrumentos de natureza psicopedagógica e didática com o objetivo de favorecer, nos contextos educativos, a elaboração pessoal destas relações, particularmente junto dos adolescentes e jovens (García, 2008).

Importa conhecer a qualidade dos relacionamentos amorosos que os jovens estabelecem de forma a otimizar os mesmos, convertendo-os numa oportunidade e num contexto promotor do seu desenvolvimento pessoal e relacional. Ao nível da intervenção será também premente a promoção de competências e de recursos pessoais que permitam assegurar o bem-estar pessoal, transformando as relações amorosas em realidades o mais positivas possível, promotoras da vivência e experienciação dos sentimentos pelo outro e pela relação de intimidade e de compromisso, com vista à satisfação da díade.

Tendo subjacente a importância das relações amorosas que os jovens estabelecem e as implicações nos diferentes domínios e não havendo um instrumento validado para a população portuguesa que permitisse avaliar estes aspetos, o presente trabalho tem como objetivos adaptar e validar para a língua portuguesa o Relationship Questionnaire (Questionário do relacionamento amoroso - QRA) e determinar a qualidade de relacionamento amoroso em jovens portugueses.

\section{Metodologia}

\section{Populaçáo e amostra}

Para a concretização dos objetivos foi desenvolvido um estudo metodológico envolvendo 127 estudantes que frequentavam uma instituição de ensino superior da região centro de Portugal, no ano letivo 2010/2011. Para a seleção da amostra não probabilística intencional definiram-se os seguintes critérios de inclusão: ter idade igual ou superior a 18 anos; ter ou ter tido namorado(a) e não ser casado.

Sendo o instrumento a validar constituído por 22 itens, a amostra mínima necessária seria de 110 adolescentes cumprindo os pressupostos referenciados por Pestana e Gageiro (2005), que 
salientam que o mínimo de respostas válidas $(\mathrm{N})$ por variável (K) é: 5x 22.

\section{Instrumento}

Foi utilizado um questionário composto por dois grupos de variáveis: características sociodemográficas (sexo, idade) e de relacionamento amoroso (ter namorado, tipo de relacionamento, componente sexual no relacionamento, frequência de contacto e futuro do relacionamento), e o Questionário do Relacionamento Amoroso (QRA).

O QRA é constituído por 22 perguntas com três opções de resposta (sim, não e às vezes) destinado a avaliar a existência de sinais indicadores de um relacionamento não saudável (National Resource Center on Domestic Violence, n.d).

No processo de adaptação cultural que corresponde à validação linguística e conceptual seguimos as guidelines internacionais (Rahman, Waheed, \& Hussain, 2003) que a seguir se descrevem.

\section{Equivalência linguística e conceptual do QRA}

A equivalência linguística e conceptual corresponde à adaptação transcultural do instrumento, tendo sido realizada conforme o método preconizado pela literatura.

A tradução de inglês para português do RQ foi efetuada por dois portugueses fluentes em inglês e conhecedores dos objetivos do estudo. Durante o período de tradução foram realizados alguns contactos com os tradutores a fim de lhes ser explicado o objetivo do instrumento de medida e as intenções subjacentes à conceção de cada item para que a tradução mantivesse o mesmo significado da versão original (equivalência do item).

A análise das diferenças entre as duas versões das traduções foi realizada por três peritos da área e da língua, resultando a primeira versão do RQ em Português (Portugal).

A retroversão ou retro tradução da primeira versão em Português (Portugal) foi realizada por outros dois tradutores, sem conhecimento prévio do questionário original.

Após a análise e comparação de todas as versões, não tendo sido encontradas discrepâncias significativas, efetuou-se o ajuste do instrumento, do qual resultou a segunda versão do RQ em Português (Portugal).

A constituição de uma comissão de peritos e submissão dos instrumentos à sua análise, teve como objetivo validar a existência dos conceitos na cultura da população alvo e, ao existirem, verificar se eram interpretados de modo semelhante nas duas culturas. Foi ainda solicitado aos juízes a revisão da apresentação dos instrumentos, as instruções para o seu preenchimento e a escala de respostas, de forma a assegurar a sua reprodutibilidade. Durante este processo foi sugerido que as perguntas tivessem resposta do tipo Likert com 4 pontos, variando de 1 (nunca) a 4 (frequentemente), pois a fidedignidade de uma escala aumenta consideravelmente a partir de duas opções de resposta, sendo mais fácil discriminar as opções de resposta. Pontuações mais elevadas correspondem a relacionamentos amorosos menos saudáveis.

A etapa seguinte consistiu na realização da reflexão falada (Thinking aloud). Aplicámos a $3^{\mathrm{a}}$ versão em Português (Portugal) dos instrumentos a um grupo com características semelhantes à população em estudo. A amostra foi constituída por seis estudantes que aceitaram voluntariamente responder aos questionários e contribuir com comentários para a sua aferição.

Este procedimento teve como objetivos testar junto da população alvo o formato e aparência visual; a compreensão das instruções e dos diferentes itens; e a recetividade e adesão aos conteúdos. Averiguámos ainda se o instrumento estava redigido com clareza, sem tendenciosidade, se solicitava o tipo de informação pretendida e se a apresentação permitia um correto preenchimento. Não houve sugestões de alterações.

\section{Equivalência psicométrica do QRA}

A equivalência psicométrica refere-se ao grau ou extensão em que as propriedades psicométricas das versões do mesmo instrumento são semelhantes. De salientar, contudo, que em virtude do original não se configurar como uma escala não foi submetido a procedimentos estatísticos, pelo que optámos por realizar os procedimentos indicados por Streiner e Norman (2008) e que se descrevem no capítulo dos resultados.

\section{Procedimentos formais e éticos e tratamento de dados}

Após receber a autorização formal para a adaptação cultural e linguística da versão Portuguesa do RQ e do 
processo da tradução e retro tradução estar finalizado, foi solicitada autorização à direção da instituição do ensino superior para a realização do estudo. Após a sua autorização, o questionário foi aplicado em contexto de sala de aula após o consentimento informado dos inquiridos.

Após a recolha dos dados, procedeu-se ao lançamento e processamento dos mesmos no programa de análise estatística de dados Statistical Package for Social Sciences (SPSS), versão 17.

As propriedades psicométricas do instrumento estudado foram calculadas utilizando medidas descritivas de resumo, correlações de cada item com o total (excluindo o respetivo item) e o alfa de Cronbach como medida de fidelidade interna dos instrumentos. A validade de constructo foi avaliada através da análise fatorial em componentes principais com rotação ortogonal pelo método varimax. Foi ainda utilizado o Teste $t$ de Student para verificar a existência de diferenças significativas entre as médias de resultados do sexo masculino e feminino.

$\mathrm{Na}$ totalidade dos itens da escala, a percentagem de não respostas foi inferior a 3\% pelo que para efeitos de tratamento estatístico, as não respostas foram substituídas pelo valor médio dos casos válidos da variável (Pestana \& Gageiro, 2005).

\section{Resultados}

\section{Características sociodemográficas e do relacionamento da amostra}

A média de idades dos estudantes é de 19,6 anos $(\mathrm{DP}=3,8)$, variando entre os 18 e os 27 anos e $78,7 \%$ (100) dos participantes são do sexo feminino. Todos os estudantes frequentam o $1^{\circ}$ ano do ensino superior e $8,7 \%$ são estudantes trabalhadores. No momento da recolha de dados, 36\% não tem namorado e dos 101 participantes que responderam à questão do tipo de relacionamento que tinham ou têm com o namorado, verificamos que $82,2 \%$ referiram que tinham/têm um relacionamento estável. Dos participantes, 45\% referiram que contactam várias vezes por semana com o namorado e 55,1\% acreditam que o futuro do relacionamento passa por viverem juntos. Em 87,9\% a relação de namoro envolve uma componente sexual. A duração de namoro dos 122 inquiridos que responderam a esta questão é em média de 17,4 meses $(\mathrm{DP}=17,4)$, variando entre os 1 e os 84 meses.

Tabela 1

Distribuição da amostra quanto às características do relacionamento*

\begin{tabular}{|c|c|c|c|}
\hline Características do relacionamento & & $\mathrm{N}^{\mathrm{O}}$ & $\%$ \\
\hline \multirow{2}{*}{ Neste momento tem namorado (a) $(n=125)$} & Sim & 80 & 64,0 \\
\hline & Não & 45 & 36,0 \\
\hline \multirow{3}{*}{ Qual é (era) o tipo de relacionamento $(\mathrm{N}=101)$} & Recente & 12 & 11,9 \\
\hline & Sem compromisso & 6 & 5,9 \\
\hline & Estável & 83 & 82,2 \\
\hline \multirow{7}{*}{$\begin{array}{l}\text { Frequência com que está (ou esteve) em contacto com o } \\
\text { seu namorado (a) }(n=80)\end{array}$} & Menos de uma vez por mês & 2 & 2,5 \\
\hline & Uma vez por mês & 1 & 1,3 \\
\hline & $15 \mathrm{em} 15$ dias & 7 & 8,8 \\
\hline & 1 vez por semana & 14 & 17,5 \\
\hline & Várias vezes por semana & 36 & 45,0 \\
\hline & 1 vez por dia & 7 & 8,8 \\
\hline & Várias vezes por dia & 13 & 16,3 \\
\hline \multirow{3}{*}{ Futuro deste namoro $(n=69)$} & Casar & 26 & 37,7 \\
\hline & Viver junto & 38 & 55,1 \\
\hline & Acabar namoro & 5 & 7,2 \\
\hline \multirow{2}{*}{ Componente sexual na relação de namoro $(n=99)$} & Sim & 87 & 87,9 \\
\hline & Não & 12 & 12,1 \\
\hline
\end{tabular}

*apesar da amostra ser constituída por 127 inquiridos, nem todos responderam a todas as questões

\section{Características psicométricas do QRA}

As características psicométricas foram determinadas por uma avaliação de fidelidade e validade, tendo deste estudo resultado uma escala final constituída por 16 itens. O estudo dos itens e da fidelidade foi realizado de acordo com os seguintes critérios: 
determinação do coeficiente de correlação entre as diversas questões e a nota global e determinação do coeficiente $(\alpha)$ alfa de Cronbach, tanto para a globalidade dos itens como para o conjunto da escala, à medida que foram sendo excluídos, um a um, os vários itens.

Tendo presente estes critérios verificamos que as correlações do item-total obtidas foram todas positivas, bastante elevadas e altamente significativas $(p<0,001)$. Todos os valores de correlação foram superiores a 0,30 . Verificam-se correlações corrigidas de cada item com o total da escala entre 0,302 e 0,743 , e a média das correlações entre todos os itens da escala atesta a homogeneidade do conjunto de enunciados (Streiner \& Norman, 2008).

Quanto ao coeficiente de alfa $(\alpha)$ de Cronbach, verifica-se que na quase totalidade (exceção do item 1) dos itens este valor desce quando ele é excluído, o que significa que melhoram a homogeneidade da escala quando estão presentes. $O$ item 1 não foi excluído pois, ao ser eliminado não melhorava significativamente o alfa de Cronbach e em termos teóricos é importante para a escala. Nenhum dos itens apresenta valores inferiores a 0,80 . Para a globalidade dos itens o valor alfa de Cronbach é de 0,847 (tabela 2) o que segundo vários autores corresponde a um bom valor (Pestana \& Gageiro, 2005; Streiner \&Norman, 2008).

\section{Tabela 2}

Estatísticas de homogeneidade dos itens e coeficientes de consistência interna ( $\alpha$ de Cronbach) do QRA

\begin{tabular}{|c|c|c|c|c|}
\hline Item & Média & $\mathrm{DP}$ & $\mathrm{r}$ corrigido & $\alpha$ de Cronbach \\
\hline 1. O seu (sua) namorado(a) troça de si de forma a magoá-la/o & 1,59 & 1,021 & 302 &, 854 \\
\hline 2. Ele/ela tem ciúmes dos seus amigos & 2,13 & ,979 &, 566 &, 833 \\
\hline 3. Ele/ela ignora as suas opiniões e interesses & 1,47 & ,682 &, 531 &, 835 \\
\hline 4. Ele/ela controla-a/o & 1,70 & ,915 &, 743 &, 819 \\
\hline 5. Ele/ela acusa-a/o de seduzir outras pessoas & 1,31 &, 668 & 667 & 827 \\
\hline 6. Ele/ela diz-lhe o que vestir ou com quem conviver & 1,32 & 684 & ,491 & 837 \\
\hline $\begin{array}{l}\text { 7. Ele/ela insiste em passar todo o tempo que têm livre juntos e zanga-se } \\
\text { quando quer fazer outras coisas }\end{array}$ & 1,64 &, 855 & ,603 &, 830 \\
\hline $\begin{array}{l}\text { 8. Ele/ela alguma vez lhe disse que mais ninguém a/o quer como } \\
\text { namorada//a }\end{array}$ & 1,23 & ,659 & ,458 &, 839 \\
\hline 9. Ele/ela toma todas as decisões & 1,23 &, 527 &, 380 &, 842 \\
\hline 10. Ele/ela insulta-a/o, humilha-a/o ou chama-lhe nomes & 1,08 & ,296 &, 400 &, 844 \\
\hline 11. Ele/ela culpa-a/o por ter de ser mau/má para si na relação & 1,17 & 463 &, 576 & 836 \\
\hline 12. Tem medo dele/a & 1,07 & 419 & 402 &, 842 \\
\hline 13. Ele/ela alguma vez a/o pressionou para ter relações sexuais & 1,14 &, 505 &, 500 &, 838 \\
\hline 14. Ele/ela alguma vez destruiu alguma coisa que era seu & 1,07 &, 311 &, 333 & 845 \\
\hline 15. Alguma vez (ainda que só uma vez) ele/ela the bateu & 1,04 & 231 & 453 & 844 \\
\hline $\begin{array}{l}\text { 16. Ele/ela tornou-se muito rapidamente demasiado sério/a em relação ao } \\
\text { vosso relacionamento }\end{array}$ & 1,75 & 1,008 & 465 &, 841 \\
\hline$\alpha$ de Cronbach & & & 847 & \\
\hline
\end{tabular}

\section{Validade}

Para o estudo da validade do instrumento e, mais especificamente, da sua estrutura interna, realizámos procedimentos de análise fatorial em componentes principais. No sentido de se maximizar a saturação dos itens, procedemos à rotação ortogonal pelo método de Varimax.

Após sucessivas análises e de acordo com critérios estatísticos e de interpretabilidade, optámos por uma estrutura fatorial de componentes principais, segundo a regra de Kaiser. Para se obter uma estrutura fatorial harmoniosa e fiável utilizaram-se, para a eliminação de itens, os seguintes critérios: saturação inferior ou igual a 0,3 ; e correlação simultânea com dois fatores, sendo que a distância entre ambos os valores não dista mais do que 0,1 .

De acordo com estes critérios, os resultados dessa análise revelaram que os 16 itens se organizam em quatro fatores que explicam 63,865\% de variância (Tabela 3).

$\mathrm{Na}$ Tabela 3 podemos igualmente observar que foi rejeitada a hipótese da matriz de correlação constituir uma matriz de identidade $\left(\chi^{2}=840,544 ; p<, 000\right)$ e 
que a medida de Kaiser-Meyer-Olkin $(\mathrm{KMO}=0,815)$ se aproxima da unidade, garantindo que a adequação do modelo fatorial a esta matriz de correlações é elevada. De salientar que o valor mais baixo de cumunalidades é de 0,47.
A designação atribuída a cada fator procurou refletir o conteúdo conceptual global dos itens que o compõem: relação de controlo; relação possessiva; relação destrutiva e relação depreciativa

Tabela 3

Matriz de componentes principais após rotação varimax, dos 16 itens do QRA

\begin{tabular}{|c|c|c|c|c|c|}
\hline & $h^{2}$ & $\mathrm{~F} 1$ & F2 & F3 & F4 \\
\hline 2. Ele/ela tem ciúmes dos seus amigos & ,590 & & ,668 & & \\
\hline 4. Ele/ela controla-a/o & ,720 & & ,675 & & \\
\hline $\begin{array}{l}\text { 7. Ele/ela insiste em passar todo o tempo que têm livre juntos e } \\
\text { zanga-se quando quer fazer outras coisas }\end{array}$ & ,656 & &, 758 & & \\
\hline 9. Ele/ela toma todas as decisões &, 571 & &, 465 & & \\
\hline $\begin{array}{l}\text { 16. Ele/ela tornou-se muito rapidamente demasiado sério/a em } \\
\text { relação ao vosso relacionamento }\end{array}$ & ,619 & &, 768 & & \\
\hline 5. Ele/ela acusa-a/o de seduzir outras pessoas & ,687 & ,699 & & & \\
\hline 6. Ele/ela diz-lhe o que vestir ou com quem conviver &, 524 &, 556 & & & \\
\hline 11. Ele/ela culpa-a/o por ter de ser mau/má para si na relação & ,686 & ,768 & & & \\
\hline 12. Tem medo dele/a &, 549 & 608 & & & \\
\hline 13. Ele/ela alguma vez a/o pressionou para ter relações sexuais & ,758 &, 831 & & & \\
\hline 1. O seu (sua) namorado(a) troça de si de forma a magoá-la/o &, 560 & & & & ,736 \\
\hline 3. Ele/ela ignora as suas opiniões e interesses & ,705 & & & &, 743 \\
\hline $\begin{array}{l}\text { 8. Ele/ela alguma vez lhe disse que mais ninguém a/o quer como } \\
\text { namorada/o }\end{array}$ &, 470 & & & & ,600 \\
\hline 10. Ele/ela insulta-a/o, humilha-a/o ou chama-lhe nomes & ,680 & & & ,694 & \\
\hline 15. Alguma vez (ainda que só uma vez) ele/ela lhe bateu & ,778 & & & ,816 & \\
\hline 14. Ele/ela alguma vez destruiu alguma coisa que era seu & ,663 & & & ,791 & \\
\hline \% variância por fator & & & $\begin{array}{l}18,19 \\
17,90 \\
14,72\end{array}$ & & 13,02 \\
\hline \% variância explicada & & & 63,85 & & \\
\hline Kaiser-Meyer-Olkin & & &, 815 & & \\
\hline Bartlett's Test of Sphericity & \multicolumn{5}{|c|}{840,$544 ; \mathrm{p}<, 0001$} \\
\hline
\end{tabular}

F1 = Relação de Controlo; F2 = Relação Possessiva; F3 = Relação Destrutiva; F4= Relação Depreciativa

O Gráfico de Cattell ou Scree Plot do QRA (Gráfico 1) permite confirmar a adequação da matriz fatorial aos dados analisados, verificando-se uma inclinação da reta até ao quarto componente, sendo este o valor correspondente a um maior afastamento entre os valores próprios (Pestana \& Gageiro, 2005).

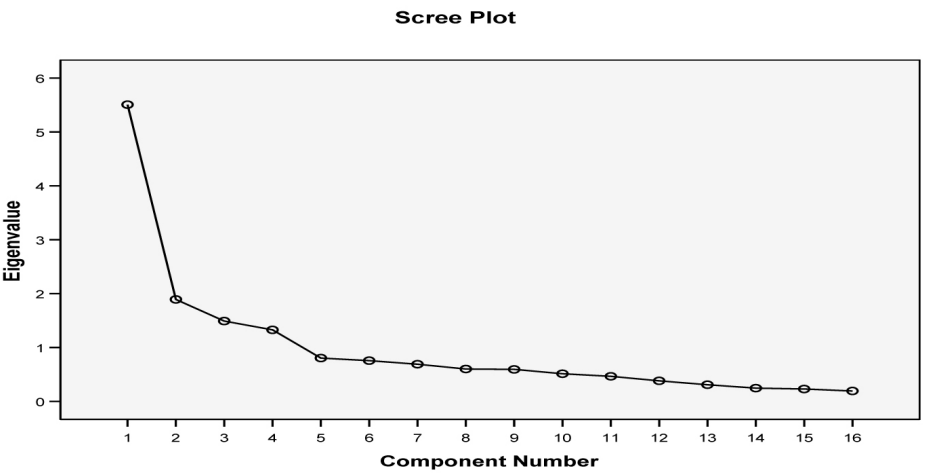

Figura 1. Gráfico de Cattell ou Scree Plot do QRA. 
Pelos dados apresentados na Tabela 4 verifica-se uma correlação entre fraca $(0,274)$ e elevada $(0,825)$ entre as várias subescalas e entre estas e o total do QRA.

Tabela 4

Correlação de Pearson entre as várias subescalas e o total do QRA
São igualmente correlações positivas e significativas $(p \leq 0,01)$, indicando que medem o mesmo constructo.

\begin{tabular}{lccccc}
\cline { 2 - 6 } & $\begin{array}{c}\text { Relaçãa } \\
\text { possessiva }\end{array}$ & $\begin{array}{c}\text { Relação de } \\
\text { controlo }\end{array}$ & $\begin{array}{c}\text { Relação } \\
\text { depreciativa }\end{array}$ & $\begin{array}{c}\text { Relação } \\
\text { destrutiva }\end{array}$ & Total do QRA \\
\hline Relação possessiva & & 0,551 & 0,413 & 0,274 & 0,825 \\
Relação de controlo & 0,551 & & 0,362 & 0,399 & 0,753 \\
Relação depreciativa & 0,413 & 0,362 & & 0,376 & 0,769 \\
Relação destrutiva & 0,274 & 0,399 & 0,376 & & 0,559 \\
Total do QRA & 0,825 & 0,753 & 0,769 & 0,559 & \\
\hline
\end{tabular}

\section{Qualidade do relacionamento amoroso}

Pela análise da Tabela 5, constatamos que, ao nível da qualidade da relação amorosa, os inquiridos do sexo masculino apresentam, em média, uma pontuação mais elevada que os do sexo feminino, quer para o total da escala quer para os diferentes fatores. Isto corresponde a relacionamentos amorosos menos saudáveis, sendo que essas diferenças têm significado estatístico ao nível da totalidade da qualidade do relacionamento amoroso $(t=2,338 ; p=0,021)$, ao nível da relação de controlo $(t=-3,063 ; p=0,003)$ e ao nível da relação destrutiva $(t=2,302 ; p=0,023)$.

Tabela 5

Média e desvio-padrão obtidos no QRA em função do sexo, e resultado do teste t de Student

\begin{tabular}{lcccccc}
\hline & Feminino & \multicolumn{3}{c}{ Masculino } & \multirow{2}{*}{ t } & p \\
\cline { 2 - 5 } & $\mathrm{M}$ & $\mathrm{DP}$ & $\mathrm{M}$ & $\mathrm{DP}$ & & \\
\hline Qualidade do relacionamento amoroso & 1,31 & 0,31 & 1,49 & 0,47 & 2,338 &, 021 \\
Relação possessiva & 1,66 & 0,64 & 1,81 & 0,65 & 1,075 &, 284 \\
Relação de controlo & 1,15 & 0,28 & 1,41 & 0,68 & 3,063 &, 003 \\
Relação depreciativa & 1,40 & 0,55 & 1,58 & 0,74 & 1,412 &, 160 \\
Relação destrutiva & 1,04 & 0,16 & 1,16 & 0,40 & 2,302 &, 023 \\
\hline
\end{tabular}

\section{Discussão}

Este estudo teve como objetivos adaptar e validar para a língua portuguesa o QRA (Questionário do relacionamento amoroso) e determinar a qualidade de relacionamento amoroso em jovens portugueses. Participaram, neste estudo, 127 estudantes de uma instituição do centro de Portugal que frequentavam o ensino superior no ano letivo 2010/2011. Em média, os estudantes tinham 19,6 anos ( $\mathrm{DP}=3,8)$, eram maioritariamente do sexo feminino (78\%) e não tinham namorado (36\%). Relativamente ao tipo de relacionamento que tinham ou tiveram com o namorado, dos participantes que responderam à questão (101), a maioria $(82,2 \%)$ referiu ter ou ter tido um relacionamento estável, contactando o namorado várias vezes por semana (45\%). Além disso, 87,9\% acreditam que o futuro do relacionamento passa por viverem juntos. Estes resultados estão em consonância com o apontado por alguns autores (Adams et al., 2001) que referem diferenças nas relações românticas entre os adolescentes em função da idade. Assim sendo as fases iniciais são caracterizadas por níveis elevados de afiliação, como a procura de proximidade física, partilha de atividades e companheirismo (Adams et al., 2001). As fases finais caracterizamse pela procura de proximidade emocional, sendo esta manifestada através da interdependência, da reciprocidade e da diversidade de atividades entre a díade e de interação social diária (Adams et al., 2001; Pinto, 2009). A maioria $(87,9 \%$ ) refere que o namoro envolve a componente sexual.

Em virtude deste instrumento não ter ainda sido submetido a nenhum estudo psicométrico não é 
possível fazer comparações. No entanto, é possível salientar que o questionário resultante deste estudo apresenta boas características psicométricas. Apresenta uma boa consistência interna ( $\alpha$ de Cronbach de 0,847), sendo o valor alfa de Cronbach para a globalidade dos itens considerado um bom valor (Pestana \& Gageiro, 2005; Streiner \& Norman, 2008). Nenhum dos itens apresenta valores inferiores a 0,80 .

Os resultados apontam para uma escala de 16 itens, distribuídos por 4 fatores (Relação possessiva, Relação de controlo, Relação depreciativa e Relação destrutiva), que explicam 63,850\% da variância.

No que respeita à qualidade da relação amorosa, os dados obtidos revelam que os relacionamentos amorosos dos inquiridos, tomando como valor de referência a mediana, são saudáveis. O indicador com resultados menos saudáveis diz respeito ao item "Ele/ ela tem ciúmes dos seus amigos" ( $\mathrm{M}=2,1 ; \mathrm{DP}=0,97)$ o que vai ao encontro do referido por Almeida et al. (2008) ao salientar que nas relações amorosas o ciúme romântico tende a estar presente podendo mesmo atingir formas doentias.

Nesta amostra, os resultados evidenciam a inexistência de violência nas relações amorosas, apesar dos estudos referirem ser um fenómeno frequente e habitual e que se inicia com frequência nas relações de namoro (Muñoz-Rivas et al., 2007). A qualidade das relações amorosas poderá ter repercussões positivas do ponto de vista do desenvolvimento destes jovens, indo ao encontro do mencionado por Pinto (2009) e ser fator protetor da vitimização e da solidão (Woodhouse et al., 2012).

No entanto, atendendo a que pontuações mais elevadas correspondem a relacionamentos amorosos menos saudáveis, os jovens do sexo masculino estabelecem, em média, relações menos positivas que os do sexo feminino, uma vez que apresentam pontuações mais elevadas, quer para o total da escala quer para as diferentes dimensões. Assim, apesar de a relação amorosa dever traduzir-se em entendimento e compreensão entre o par romântico, em que cada um mantém a sua própria identidade e deseja fazer o bem à pessoa amada sem esperar recompensa (Rosset, 2004), as relações positivas nem sempre acontecem. Verificamos ainda que as diferenças, em função do sexo, têm significado estatístico ao nível da totalidade da qualidade do relacionamento amoroso, da relação de controlo e da relação destrutiva. Estes resultados vêm ao encontro do referido por Shulman e Kipnis (2001), ao mencionarem que nem sempre as relações de namoro se processam de modo positivo e gratificante. Por outro lado, os estudos evidenciam (Almeida et al., 2008; Arnocky et al., 2012) que as relações amorosas mediadas pelo ciúme, controlo e posse afetam o desenvolvimento interpessoal dos jovens. Nesta amostra, a dimensão relação possessiva é a que apresenta pontuações mais altas, em ambos os grupos.

Numa perspetiva de prevenção das relações de violência e de relações dominantes e vitimizadoras, torna-se importante o desenvolvimento de instrumentos de natureza psicopedagógica e didática que permitam uma intervenção junto dos adolescentes e jovens, numa perspetiva desenvolvimental, especialmente em contextos educativos, tal como recomenda García (2008).

Assim, os resultados obtidos na adaptação do QRA à população portuguesa confirmam este instrumento como rigoroso, válido e com boas características psicométricas. A escala constituída por 16 itens, distribuídos por 4 fatores (Relação possessiva, Relação de controlo, Relação depreciativa e Relação destrutiva) que explicam 63,850\% da variância, apresenta uma boa consistência interna ( $\alpha$ de Cronbach de 0,847). Isto permite a possibilidade da escala ser usada com confiança em estudos futuros na avaliação da relação amorosa dos adolescentes, assim como na prática educacional com o objetivo de avaliar a eficácia de intervenções junto desta população.

Sugerimos que em estudos futuros a amostra seja maior e colhida em diversos pontos do país.

Em síntese, consideramos que este estudo pode contribuir para conhecer a qualidade dos relacionamentos amorosos dos jovens de forma a otimizá-los com vista ao seu desenvolvimento pessoal e relacional.

\section{Conclusão}

Os estudos recentes demonstram que os relacionamentos amorosos assumem um significado especial na adolescência e juventude, constatando-se a existência de associações entre estes e aspetos do desenvolvimento individual.

Os resultados apontam para uma escala com uma boa consistência interna e confiabilidade, confirmando 
o instrumento como rigoroso, válido, com boas características psicométricas. Assim, poderá ser utilizado em estudos futuros, sendo muito importante para a prática de enfermagem na área do adolescente e jovem.

A validação do Relashionship Questionnaire (RQ) para a população portuguesa constitui, assim, um valor acrescentado aos instrumentos de investigação direcionados para jovens, dado que possibilita determinar a qualidade dos seus relacionamentos amorosos e implementar programas de intervenção com vista ao desenvolvimento de competências pessoais e relacionais que potenciem o estabelecimento de relações íntimas saudáveis, responsáveis e gratificantes.

\section{Referências bibliográficas}

Adams, R., Laursen, B., \& Wilder, D. (2001). Characteristics of closeness in adolescent romantic relationships. Journal of Adolescence, 24(3), 353-363. doi:10.1006/jado.2000.0402

Almeida, T., Rodrigues, K., \& Silva, A. (2008). O ciúme romântico e os relacionamentos amorosos heterossexuais contemporâneos. Estudos de Psicologia, 13(1), 83-90. doi:10.1509/S1413-294X2008000100010

Arnocky, S., Sunderani, S., Miller, J., \& Vaillancourt, S. (2012). Jealousy mediates the relationship between attractiveness comparison and females' indirect aggression. Personal Relationships, 19(2), 290-303. doi:10.1111/j.1475-6811.2011. 01362.X

Collins, W., Welsh, D., \& Furman, W. (2009). Adolescent romantic relationships. Annual Review Psychology, 60, 631-652.

Connolly, J., \& McIsaac, C. (2011). Romantic relationship in adolescence. In M. K. Underwood \& L. H. Rosen (Eds.), Social development: Relationship in infancy, childhood, and adolescence (pp.180-206). New York, NY: Guilford.

Fiol, E. (Coord.). (2007). Del mito del amor romántico a la violencia contra las mujeres en la pareja. Palma, España: Universidad de les Illes Balears.

García, A. (Coord.). (2008). Concepciones acerca de las relaciones de amor y el conocimiento de las necesidades del otro u otra: Un estúdio con adolescentes y jóvenes con objeto de entender y prevenir el maltrato en las relaciones de pareja. Barcelona, España: Universidad Autònoma de Barcelona.
Hand, L., \& Furman, W. (2009). Rewards and costs in adolescent other-sex friendships: Comparisons to same-sex friendships and romantic relationships. Social Development, 18(2), 270287. doi:10.111/j.1467-9807.2008.00499.x

Madsen, S., \& Collins, W. (2011). The salience of adolescent romantic experiences for romantic relationship qualities in young adulthood. Journal of Research on Adolescence, 21(4), 789-801. doi:10.111/j.1532-7795.2011.00737.x

Markiewicz, D., Lawford, H., Doyle, A., \& Haggart, N. (2006). Developmental differences in adolescents' and young adults' use of mothers, fathers, best friends, and romantic partners to fulfil attachment needs. Journal of Youth and Adolescence, 35(1), 121-134. doi:10.107/s10964-005-9014-5

Muñoz-Rivas, M., Gómez, J., O’Leary, K., \& Lozano, P. (2007). Physical and psychological aggression in dating relationships in Spanish university students. Psicothema, 19(1), 102-110.

National Resource Center on Domestic Violence. (n.d.). Community action kit: Helping preteens and teens build healthy relationships. Recuperado de http://www.vawnet. org/advanced-search/summary.php?doc_id=2255\&find_ type $=$ web_desc_GC

Pestana, M., \& Gageiro, J. (2005). Análise de dados para ciências sociais. A complementaridade do SPSS ( $4^{\mathrm{a}}$ ed). Lisboa, Portugal: Sílabo.

Pinto, M. (2009). Intimidade em adolescentes de diferentes grupos étnicos. Lisboa, Portugal: ACIDI.

Rahman, A., Waheed, W., \& Hussain, N. (2003). Translation and cultural adaptation of health questionnaires. Journal of Pakistan Medical Association, 53(4), 142-147.

Rosset, S. (2004). O casal nosso de cada dia. Curitiba, Brasil: Sol.

Shulman, S., \& Kipnis, O. (2001). Adolescent romantic relationships: A look from the future. Journal of Adolescence. 24(3), 337-351. doi:10.1006/jado.2001.0409

Starr, L., Davila, J., Stroud, C., Clara Li, P., Yoneda, A., Hershenberg, R., \& Ramsay, M. M. (2012). Love hurts (in more ways than one): Specificity of psychological symptoms as predictors and consequences of romantic activity among early adolescent girls. Journal of Clinical Psychology, 68(4), 373381. doi:10.1002/jclp.20862

Streiner, D., \& Norman, G. (2008). Health and measurement scales. A practical guide for their development and use (4th ed.). Oxford, England: Oxford University Press.

Woodhouse, S. S., Dykas, M., \& Cassidy, J. (2012). Loneliness and peer relations adolescence. Social Development, 21(2), 273293. doi:10.1111/j.1467-9507.2011.00611.x 